\section{(A) Check for updates}

Cite this: Food Funct., 2020, 11, 3749

DOI: $10.1039 /$ d0fo90012e

rsc.li/food-function

\title{
Correction: 5-Heptadecylresorcinol attenuates oxidative damage and mitochondria-mediated apoptosis through activation of the SIRT3/FOXO3a signaling pathway in neurocytes
}

\author{
Jie Liu, ${ }^{a}$ Yu Wang, ${ }^{a}$ Yiming Hao, ${ }^{a}$ Zongwei Wang, ${ }^{a}$ Zihui Yang, ${ }^{a}$ Ziyuan Wang ${ }^{a}$ and \\ Jing Wang*a,b
}

Correction for '5-Heptadecylresorcinol attenuates oxidative damage and mitochondria-mediated apoptosis through activation of the SIRT3/FOXO3a signaling pathway in neurocytes' by Jie Liu et al., Food Funct., 2020, DOI: 10.1039/c9fo03028j.

The authors regret the incorrect version of Fig. 4 was included in the original article. The correct version of Fig. 4 is presented below.

A

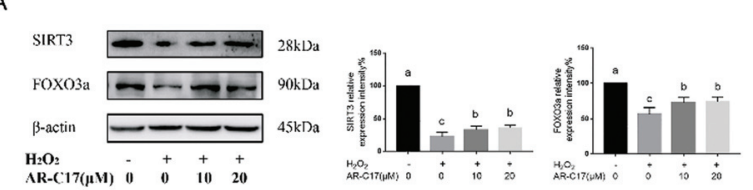

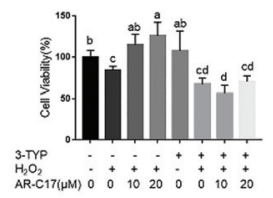

C
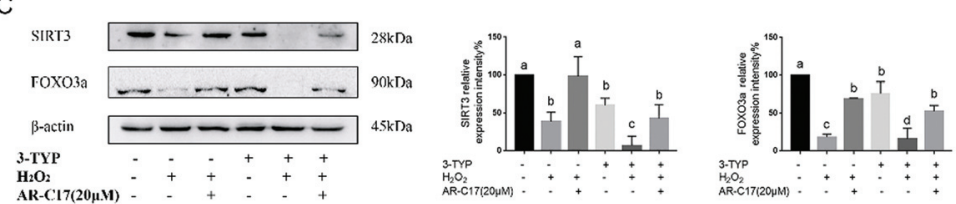

D
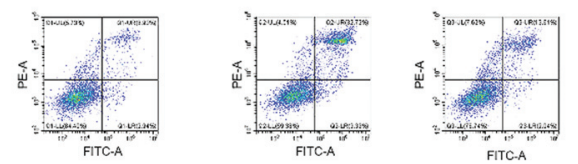

$\mathrm{NC}$
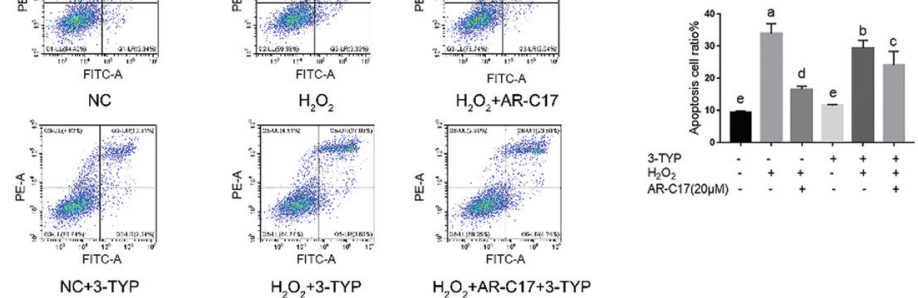

Fig. 4 AR-C17 prevents oxidative damage and mitochondria-mediated apoptosis through the SIRT3-FOXO3a signaling pathway. Cells were incubated with or without $30 \mu \mathrm{M}$ SIRT3 inhibitor (3-TYP) for $12 \mathrm{~h}$. They were then incubated with AR-C17 for another $48 \mathrm{~h}$, and finally exposed to $250 \mu \mathrm{M}$ $\mathrm{H}_{2} \mathrm{O}_{2}$. (A) The effect of AR-C17 on the protein expression of SIRT3 and FOXO3a. (B) The effect of AR-C17 and cotreatment with 3-TYP on cell viability. (C) The effect of AR-C17 and cotreatment with 3-TYP on protein expression of SIRT3 and FOXO3a. (D) The effect of AR-C17 and cotreatment with 3-TYP on cell apoptosis. Data are presented as the means \pm SD $(n=3)$. Results marked with the same letters are not significantly different $(P<0.05)$.

The Royal Society of Chemistry apologises for these errors and any consequent inconvenience to authors and readers.

${ }^{a}$ China-Canada Joint Lab of Food Nutrition and Health (Beijing), Beijing Technology \& Business University (BTBU), Beijing 100048, China. E-mail: wangjing@th.btbu.edu.cn ${ }^{b}$ Beijing Advanced Innovation Center for Food Nutrition and Human Health, Beijing Technology \& Business University, Beijing 100048, China 